\title{
Variability in clinical phenotype despite common chromosomal deletion in Smith-Magenis syndrome [del(17)(p11.2p11.2)]
}

Lorraine Potocki, $M D^{1,2}$, Christine J. Shaw, $B S^{1}$, Pawel Stankiewicz, $M D, P h D^{1}$, and James R. Lupski, MD, PhD ${ }^{1-3}$

\begin{abstract}
Purpose: This report delineates the phenotypic features in a cohort of 58 individuals with Smith-Magenis syndrome (SMS) and compares features of patients with the common microdeletion to those of patients with variable sized deletions, and the three previously reported patients who harbor a mutation in RAl1 (retinoic acid induced 1). Methods: From December 1990 thru September 1999, 58 persons with SMS were enrolled in a 5-day multidisciplinary clinical protocol at the General Clinical Research Center (GCRC), Texas Children's Hospital. Each patient had a cytogenetically evident deletion in 17p11.2. Results: Of the 51 patients in whom the molecular extent of the chromosomal deletion could be delineated by pulsed-field gel electrophoresis (PFGE) and/or fluorescent in situ hybridization (FISH), 39 ( $\approx 76 \%$ ) had the common SMS deletion. Smaller or larger deletions were seen in $\approx 12 \%$ and $\approx 10 \%$ of patients, respectively, and 1 patient had a complex chromosomal rearrangement including a deletion in $17 p 11.2$. Parent of origin was determined by polymorphic marker analysis in a subset of patients: maternal $\approx 43 \%$, paternal $\approx 57 \%$. All patients had impaired cognitive and adaptive functioning and had at least one objective measure of sleep disturbance. Other common features (seen in $>50 \%$ of patients) include short stature, ophthalmological, and otolaryngological anomalies, hearing impairment, abnormal EEG, and scoliosis. Cardiac and renal anomalies were seen in $\approx 45 \%$ and $\approx 19 \%$ of patients, respectively. There are no statistically significant differences in the incidence of these abnormalities in patients with the common deletion compared to those patients with smaller or larger sized deletions. Conclusions: Despite a common deletion size in $76 \%$ of patients with SMS, the only constant objectively defined features among these patients are sleep disturbances, low adaptive functioning, and mental retardation. There is no pathognomonic clinical feature, no characteristic cardiovascular defect, renal anomaly, otolaryngological or ophthalmic abnormality in SMS. Genet Med 2003:5(6): 430-434.
\end{abstract}

Key Words: microdeletion, homologous recombination, chromosome 17, Smith-Magenis syndrome

Smith-Magenis syndrome (SMS, MIM 182290) is a multiple congenital anomalies and mental retardation syndrome associated with an interstitial deletion within chromosome 17p11.2. More than 100 patients with SMS have been described. ${ }^{1}$ Clinical characteristics include minor craniofacial and skeletal anomalies such as brachycephaly, frontal bossing, synophrys, midfacial hypoplasia, short stature and brachydactyly, neurobehavioral abnormalities such as aggressive and self-injurious behavior and sleep disturbances, ophthalmic, otolaryngological, cardiac, and renal anomalies. ${ }^{1,2}$ In the majority of patients, a common deletion size is observed. ${ }^{3-5}$ This

From the Departments of ${ }^{1}$ Molecular and Human Genetics and ${ }^{3}$ Pediatrics, Baylor College of Medicine and ${ }^{2}$ Texas Children's Hospital, Houston, Texas.

Lorraine Potocki, MD, FACMG, Assistant Professor, Department of Molecular and Human Genetics, Baylor College of Medicine, Texas Children's Hospital, 6621 Fannin, Mail Code: CC-1560, Houston, TX 77030.

Received: June 17, 2003.

Accepted: August 22, 2003.

DOI: 10.1097/01.GIM.0000095625.14160.AB common deletion is derived from nonallelic homologous recombination (NAHR) between low-copy repeat (LCR) gene clusters (distal and proximal SMS-REPs) during either maternal or paternal gametogenesis. 5,6 Approximately 20\% to 25\% of SMS patients reported have either smaller or larger sized deletions. ${ }^{7-9}$ In the patients with unusual sized (smaller or larger) deletions, the precise mechanism of deletion remains to be determined; however, other LCRs predominate at breakpoints. ${ }^{10}$ Three additional SMS patients without a microdeletion have been reported recently, each harbors a mutation in RAI1, which maps within the SMS critical region. ${ }^{11}$

Fifty-eight persons with SMS were evaluated through an International Review Board (IRB) approved multidisciplinary clinical protocol in the General Clinical Research Center (GCRC) at the Texas Children's Hospital in Houston. Clinical finding on a subset of these patients $(N=27)$ were reported previously, but without molecular studies to define the extent of the deletion. ${ }^{2}$ For this study, 51 of the 58 patients underwent molecular analysis to determine deletion size. Thirty-nine of 51 persons (76\%) analyzed by PFGE and/or FISH were found 
to have the common SMS deletion. Although there are common physical features among these patients, the variability between individual patients with molecularly identical sized deletions is marked. Multiple genes have been identified within the SMS common deletion interval. ${ }^{8}$ Recently, mutations in RAI1 have been identified in three patients with many SMS characteristics, yet these individuals are not affected with cardiac or renal abnormalities, short stature, midfacial hypoplasia, or brachydactyly. ${ }^{11}$ Only MYO15 has been clearly associated with a specific phenotypic feature (sensorineural hearing impairment) in a patient with SMS (1123) who harbored a recessive mutation on the nondeleted allele. ${ }^{12}$

We examined the variability of phenotype of SMS patients with the common deletion and compared that observed for patients with alternatively sized deletions. Remarkably, despite identical deletion in $76 \%$ of patients, substantial clinical variability can be observed.

\section{METHODS}

\section{Patient ascertainment and evaluation}

From December 1990 to September 1999, 58 persons with SMS [del(17)(p11.2p11.2)], (43\% male; mean age 9 years; age range 1 year 6 months to 31 years) were enrolled in the multidisciplinary clinical study of SMS through the GCRC at the Texas Children's Hospital in Houston, under a protocol approved by the Baylor College of Medicine IRB. All 58 patients were ascertained by abnormal chromosome analysis with $\operatorname{del}(17)(\mathrm{p} 11.2 \mathrm{p} 11.2)$. Informed consent was obtained from the patient's parent or legal guardian. Clinical evaluations performed in these patients include: physical examination, developmental and cognitive profiles, ophthalmological and otolaryngological examinations, audiological assessment, 12-hour or 24-hour polysomnography, echocardiogram, renal ultrasound, scoliosis survey, radiographs of forearms and hands, lipid profile, and thyroid function studies.

\section{Cytogenetic analysis}

Each patient had an interstitial deletion in $17 \mathrm{p} 11.2$ as evidenced by G-banded chromosome analysis. The presence of a deletion was confirmed by FISH analysis using probes specific for $F L I I^{13}$ and ZFP179 (ZNF179), ${ }^{14}$ both mapping within the SMS common deletion region, with the peripheral myelin protein 22 gene, PMP22, mapping within the commonly duplicated CMT1A region, ${ }^{15}$ as a control. All patients were deleted for FISH probe FLII. ZFP179 was deleted in all patients with common deletions, two patients with smaller deletions (1195 and 1354), and three patients with larger deletions (147, 536, and 1153). Large deletion patients (200 and 484) were deleted for all three probes and are likely affected with hereditary neuropathy with liability to pressure palsies (HNPP) due to the deletion of PMP22. ${ }^{16}$ The patient with the complex rearrangement (1221) was reported previously. ${ }^{17}$

\section{PFGE analysis}

PFGE was performed on the patient samples as described ${ }^{5,18}$ to identify whether the deletions represented the LCR-mediated common rearrangements. A deletion is considered common if the breakpoints map within the proximal and distal SMS-REPs and is distinguished by a unique de novo $\approx 1.1-\mathrm{Mb}$ band, corresponding to the SMS rearrangement-specific common junction fragment. The molecular extent of the deletion was not determined in 7 of the 58 patients as cell lines were not available for culture.

\section{Genotyping}

Parental origin of the deleted chromosome was determined in 30 of the 58 cases by microsatellite marker analysis using genomic DNA purified from peripheral blood as described. ${ }^{6}$ The remaining patient samples were either not analyzed for parental origin or were uninformative. The parent of origin was identified by lack of transmission of an allele to the patient for two or more of loci D17S805, D17S2256, D17S2257, D17S2259, and D17S52258.

\section{RESULTS}

A recombination-specific junction fragment of $\approx 1.1 \mathrm{Mb}$ was identified in 39 of 51 patients $(\approx 76 \%)$, suggesting the common SMS deletion (Fig. 1 ). The $\approx 1.1-\mathrm{Mb}$ junction fragment was not evident in 12 patients, six of whom (12\%) have smaller deletions (540, 641, 1190, 1195, 1354, and 1456) and five of whom (10\%) have larger deletions $(147,200,484$, 536, and 1153), as defined by FISH analyses. ${ }^{10}$ One patient (1221) has a complex chromosomal rearrangement leading to deletion of $17 \mathrm{p} 11.2 .{ }^{17}$ The deletion was of paternal origin in $17 / 30$ (56.6\%) cases and maternal in 13/30 (43.3\%).

The patients exhibited the characteristic physical features of SMS including one or more of the following: brachycephaly, frontal bossing, synophrys, midfacial hypoplasia, downturned mouth, and brachydactyly. A history of developmental delay and/or cognitive impairment was ascertained, and all patients had cognitive impairment and low adaptive functioning by objective psychometric analysis. ${ }^{19}$ The majority of patients had full-scale IQ scores in the moderate to mild range of mental retardation. ${ }^{2,19}$ Low adaptive functioning (by the Vineland Adaptive Scales) was seen in all patients, regardless of IQ score. ${ }^{19}$ All patients showed sleep disturbances when assessed by objective criteria using the Epworth Sleepiness Scale, total sleep time and sleep stage distribution (by polysomnography), and the Multiple Sleep Latency Test. ${ }^{20}$ Variable features in SMS patients with del(17)(p11.2p11.2) included otolaryngological and ophthalmological abnormalities, hearing impairment, short stature, scoliosis, electroencephalogram (EEG) abnormalities, cardiac, and renal or urinary tract anomalies. Abnormalities in lipid profiles and metacarpal phalangeal profile are also variable but have been previously reported in this cohort. ${ }^{21,22}$ 


\section{SMS Common Deletions}

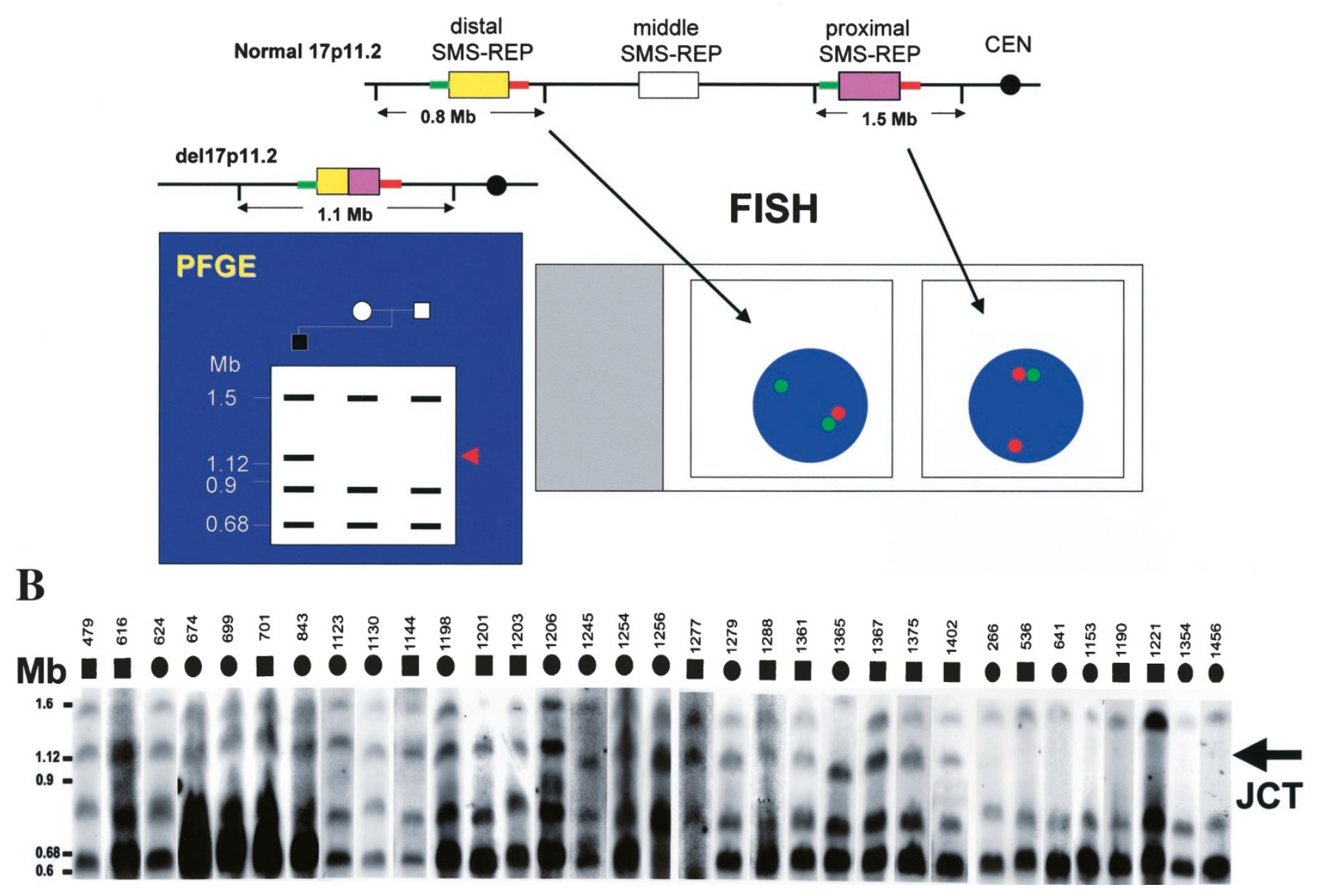

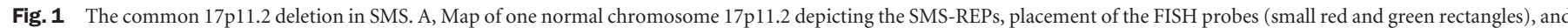

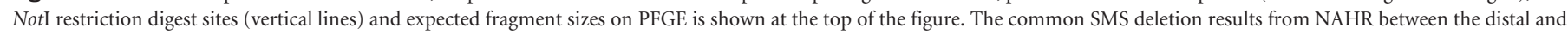

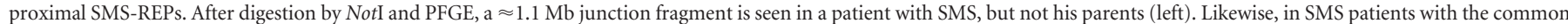

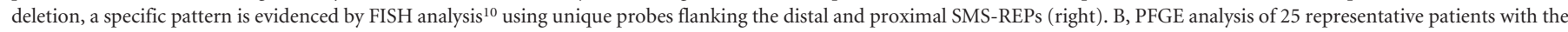

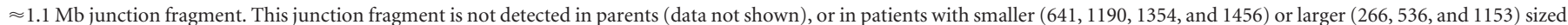
deletions, nor the patient with the complex rearrangement (1221).

Table 1 summarizes the variable clinical findings in this cohort of 58 patients. Tables 2 and 3 summarize the clinical features in 51 patients in whom deletion size was determined, and stratifies these findings based on the molecular size of the 17p11.2 deletion. Patients in this study are considered to have otolaryngological abnormalities if findings were evident on physical examination (by an otolaryngologist) at the time of the GCRC admission. Thus, a past medical history of otitis media does not qualify, but findings of tympanic scarring due to chronic otitis media, or acute otitis media do. Findings in this category include the following: cleft palate or submucous cleft palate; velopharyngeal incompetence; vocal cord polyps, nodules, paralysis, thickening, or edema; tympanic membrane perforation, scaring or active otitis media; sinusitis; cholesteatoma; laryngomalacia; and adenoid hypertrophy. Ophthalmic abnormalities are similar to those previously reported $^{23}$ and include myopia, myopic astigmatism, hyperopia, microcornea, iris dysplasia, coloboma, posterior embryotoxon, esotropia, and strabismus.
Hearing impairment in SMS may be conductive, sensorineural, or mixed. ${ }^{2}$ Fifty-seven patients underwent formal audiological evaluation. Of the patients with the common deletion, $28 \%$ had normal hearing sensitivity, $46 \%$ had conductive impairment, $13 \%$ sensorineural, and $13 \%$ mixed. Of the patients harboring a small deletion, 2 had normal sensitivity, 1 had conductive impairment, 1 sensorineural, and 2 mixed. Two patients with larger deletions had conductive impairment, 1 had mixed, and 2 had normal hearing sensitivity.

An EEG was performed as part of the polysomnography (sleep study). Seizures occur in $10 \%$ to $20 \%$ of SMS patients, ${ }^{1}$ although a higher percentage of patients were recognized to have EEG abnormalities in the absence of clinically evident seizures. ${ }^{2}$ In this study, a clinical history of seizures was associated with either normal or abnormal EEGs. Only patients with abnormal EEGs are noted in the tables. Abnormal findings include focal spike discharge activity or generalized activity, including bursts of generalized spike and wave activity. 
Table 1

Phenotypic features in patients with $\operatorname{del}(17)(\mathrm{p} 11.2 \mathrm{p} 11.2)$

\begin{tabular}{lcc}
\hline Clinical feature & Total cohort $^{a, b}$ & $\begin{array}{c}\text { Molecularly defined } \\
\text { cohort }^{a}\end{array}$ \\
\hline Otolaryngologic abnormality & $45 / 50(90 \%)$ & $40 / 45(89 \%)$ \\
Ophthalmologic abnormalities & $51 / 56(91 \%)$ & $45 / 49(92 \%)$ \\
Hearing impairment & $38 / 57(67 \%)$ & $35 / 51(69 \%)$ \\
Short stature (<5th percentile) & $38 / 58(66 \%)$ & $34 / 51(67 \%)$ \\
Scoliosis & $35 / 58(60 \%)$ & $31 / 51(61 \%)$ \\
EEG abnormalities & $31 / 58(54 \%)$ & $29 / 51(57 \%)$ \\
Cardiac anomaly & $20 / 44(45 \%)$ & $18 / 40(45 \%)$ \\
Renal/GU anomaly & $11 / 57(19 \%)$ & $9 / 50(18 \%)$ \\
\hline
\end{tabular}

${ }^{a}$ Denominators indicate the number of patients in each category studied for abnormality at our institution.

${ }^{b}$ The total cohort includes all 58 patients with del(17)(p11.2p11.2), including the 7 in which the molecular extent of the deletion was not determined.

Table 2

Phenotypic comparison in patients with variable sized deletions

\begin{tabular}{lccc}
\hline Clinical feature & $\begin{array}{c}\text { Small } \\
\text { deletion }^{a}\end{array}$ & $\begin{array}{c}\text { Large } \\
\text { deletion }^{a}\end{array}$ & $\begin{array}{c}\text { Complex } \\
\text { rearrangement }\end{array}$ \\
\hline Otolaryngologic abnormality & $4 / 5(80 \%)$ & $4 / 4(100 \%)$ & - \\
Ophthalmologic abnormalities & $5 / 6(83 \%)$ & $4 / 4(100 \%)$ & + \\
Hearing impairment & $4 / 6(67 \%)$ & $3 / 5(60 \%)$ & - \\
$\begin{array}{l}\text { Short stature }(<5 \text { th } \\
\text { percentile) }\end{array}$ & $5 / 6(83 \%)$ & $4 / 5(80 \%)$ & + \\
$\begin{array}{l}\text { Scoliosis } \\
\text { EEG abnormalities }\end{array}$ & $4 / 6(67 \%)$ & $4 / 5(80 \%)$ & + \\
Cardiac anomaly & $5 / 6(83 \%)$ & $4 / 5(80 \%)$ & + \\
Renal/GU anomaly & $3 / 5(60 \%)$ & $3 / 3(100 \%)$ & - \\
\hline
\end{tabular}

${ }^{a}$ Denominators indicate the number of patients in each category studied for abnormality at our institution.

${ }^{b}$-indicates absence, and + , presence of finding in the single patient with a complex chromosomal rearrangement.

Table 3

Clinical variance in patients with the common SMS deletion

\begin{tabular}{lc}
\hline Clinical Feature & No. Abnormal $^{a}(\%)$ \\
\hline Otolaryngologic abnormality & $32 / 35(91 \%)$ \\
Ophthalmologic abnormalities & $35 / 38(92 \%)$ \\
Hearing impairment & $28 / 39(72 \%)$ \\
Short stature (<5th percentile) & $24 / 39(62 \%)$ \\
Scoliosis & $22 / 39(56 \%)$ \\
EEG abnormalities & $19 / 39(49 \%)$ \\
Cardiac anomaly & $12 / 31(39 \%)$ \\
Renal/GU anomaly & $6 / 39(15 \%)$
\end{tabular}

${ }^{a}$ Denominators indicate the number of patients studied for abnormality at our institution.

These findings are considered epileptogenic, although were not associated with clinically evident seizures. Of the 6 patients studied with smaller deletions (not all with the same break- points), 5 have abnormal EEGs, yet only 3 have a clinical history of seizures.

The presence of cardiac anomalies was determined by $2 \mathrm{D}$ echocardiography performed on 44 of the 58 patients. Valvular abnormalities (e.g., pulmonary regurgitation and tricuspid regurgitation) with or without associated ventricular changes (e.g., right ventricular enlargement) were more common than structural defects such as patent foramen ovale, cleft mitral valve, atrial or ventricular septal defects, or (in one case) tetralogy of Fallot. These structural defects were found in only $21 \%$ of patients (9/44), and seen in patients with common, small, large, and undetermined sized deletions.

Renal ultrasound was performed on 57 patients. Genitourinary abnormalities include the following: duplication of ureters; ureteropelvic junction obstruction; renal pelvic ectasia; ectopic kidney; abnormally small kidneys, and unilateral renal agenesis. Of the 39 patients with common deletion, 6 had an abnormality including 3 patients with duplication of the ureters, 2 with abnormally small kidneys, and 1 with ureteropelvic junction obstruction.

\section{DISCUSSION}

Smith-Magenis syndrome (SMS) is a multiple congenital anomalies, mental retardation syndrome associated with a heterozygous deletion of chromosome $17 \mathrm{p} 11.2$. Characteristic physical features include minor craniofacial ${ }^{24}$ and skeletal anomalies, ${ }^{22}$ ophthalmological ${ }^{23}$ and otolaryngological abnormalities, and malformations of the heart, including tetralogy of Fallot, 25,26 and kidney. ${ }^{1,2}$ The neurobehavioral features of SMS are perhaps the most distinctive characteristic of this microdeletion syndrome and include self-injurious, aggressive, and maladaptive behavior $^{3,19,27,28}$ and significant sleep disturbances.,320,29

We have previously described the clinical phenotype of SMS in 27 patients with del(17)(p11.2p11.2), ${ }^{2}$ and herein have extensively characterized the clinical phenotype in another 31 SMS patients. Whereas common phenotypic features can be defined, a wide range of clinical variability exists among patients. Interestingly, although the majority $(76 \%)$ of the evaluated SMS patients have the same-sized deletion by molecular analysis, the clinical spectrum is variable even among these patients. Furthermore, as previously described in SMS patients with different sized deletions, there is no correlation between the size of the deletion and the major clinical features of SMS. ${ }^{7}$ With the exception of abnormalities found on EEG and renal ultrasound, the patients with the smaller-sized deletions have features similar to those seen in the common deletion patients. Although the presence of EEG abnormalities does not seem to correlate with other clinical features, there does seem to be a correlation with deletion size in that the patients with the smaller deletions are more likely to have an abnormal EEG; however, the difference was not statistically significant (Fisher's Exact, $P=0.18$ ). The explanation for this observation is elusive, because genes implicated in epilepsy do not map to the breakpoints of these deletions; however, this finding may be reflective of a position effect, ${ }^{30}$ or could merely be due to prob- 
lems inherent in analyzing data from a small sample size. Our patients with smaller-sized deletions were more often affected with genitourinary anomalies than patients with the common or large deletion; however the difference was not statistically significant (Fisher's Exact, $P=0.06$ ).

SMS has been considered to be a contiguous gene deletion syndrome (CGDS), and it had been postulated that the phenotypic variation among SMS patients could be due to variations in the size of the deletion, although a common deletion region was previously assigned by polymorphic marker analysis. ${ }^{3,4}$ Recently, three patients with physical, developmental, and behavioral features of SMS were reported to have mutations of RAI1. ${ }^{11}$ Although these patients have ocular and otolaryngological abnormalities, and one is affected with seizures, they have no malformations of the cardiovascular or genitourinary systems, nor are they affected with short stature, midfacial hypoplasia, or brachydactyly. ${ }^{11}$ With the recent data establishing RAI1 mutations in persons with characteristics of SMS, it is plausible that the major features of this syndrome are due to RAI1 haploinsufficiency. This phenomenon has been observed in other multiple congenital anomaly syndromes such as Rubinstein-Taybi (RTS; MIM 180849) and Alagille (AGS; MIM 118450) syndromes resulting from haploinsufficiency of a gene encoding a transcription factor (CBP and JAG1, respectively). Although the vast majority of SMS patients reported harbor an interstitial deletion of $17 \mathrm{p} 11.2$, the proportion of patients with features of SMS and a heterozygous RAI1 mutation remains to be determined, and indeed, the full phenotypic spectrum of SMS may be evident only in persons harboring the chromosomal deletion.

Clinical evaluation and management of newly diagnosed SMS patients may eventually depend on the results of cytogenetic and molecular analyses; however, given the data presented herein, and until more data are collected on patients with RAI1 mutations, current management guidelines (www.geneclinics.org) should be followed for assessment.

\section{ACKNOWLEDGMENTS}

This work was supported in part by grants from the National Institute of Child Health and Human Development (NIH) (K08 HD01149) (L.P.) and (P01 HD39420) (J.R.L.), the Baylor College of Medicine Mental Retardation Research Center (HD2406407), and the Texas Children's Hospital General Clinical Research Center (M01RR00188). We thank the patients and their families for participation, Marjorie A. Withers and Doreen E. Osterholm for technical assistance, and Dr. O'Brien Smith for assistance with statistical analysis.

\section{References}

1. Chen K-S, Potocki L, Lupski JR. The Smith-Magenis syndrome [del(17)p11.2]: Clinical review and molecular advances. Ment Retard Dev Disabil Res Rev 1996;2: $122-129$.

2. Greenberg F, Lewis RA, Potocki L, Glaze D, Parke J, Killian J et al. Multi-disciplinary clinical study of Smith-Magenis syndrome (deletion 17p11.2). Am J Med Genet 1996;62:247-254

3. Greenberg F, Guzzetta V, Montes de Oca-Luna R, Magenis RE, Smith AC, Richter SF et al. Molecular analysis of the Smith-Magenis syndrome: a possible contiguous-gene syndrome associated with del(17)(p11.2). Am J Hum Genet 1991;49:1207-1218.
4. Juyal RC, Figuera LE, Hauge X, Elsea SH, Lupski JR, Greenberg F et al. Molecular analyses of 17p11.2 deletions in 62 Smith-Magenis syndrome patients. Am J Hum Genet 1996;58:998-1007.

5. Chen K-S, Manian P, Koeuth T, Potocki L, Zhao Q, Chinault AC et al. Homologous recombination of a flanking repeat gene cluster is a mechanism for a common contiguous gene deletion syndrome. Nat Genet 1997;17:154-163.

6. Shaw CJ, Bi W, Lupski JR. Genetic proof of unequal meiotic crossovers in reciprocal deletion and duplication of 17p11.2. Am J Hum Genet 2002;71:1072-1081.

7. Trask BJ, Mefford H, van den Engh G, Massa HF, Juyal RC, Potocki L et al. Quantification by flow cytometry of chromosome-17 deletions in Smith-Magenis syndrome patients. Hum Genet 1996;98:710-718.

8. Bi W, Yan J, Stankiewicz P, Park S-S, Walz K, Boerkoel CF et al. Genes in a refined Smith-Magenis syndrome critical deletion interval on chromosome 17p11.2 and the syntenic region of the mouse. Genome Res 2002;12:713-728.

9. Vlangos CN, Yim DK, Elsea SH. Refinement of the Smith-Magenis syndrome critical region to approximately $950 \mathrm{~kb}$ and assessment of $17 \mathrm{p} 11.2$ deletions: Are all deletions created equally? Mol Genet Metab 2003;79:134-141.

10. Stankiewicz P, Shaw CJ, Dapper JD, Wakui K, Shaffer LG, Withers M et al. Genome architecture catalyzes nonrecurrent chromosomal rearrangements. Am J Hum Genet 2003;72:1101-1116.

11. Slager RE, Newton TL, Vlangos CN, Finucane B, Elsea SE. Mutations in RAII associated with Smith-Magenis syndrome. Nat Genet 2003;33:466-468.

12. Liburd N, Ghosh M, Riazuddin S, Naz S, Khan S, Ahmed Z et al. Novel mutations of MYO15A associated with profound deafness in consanguineous families and moderately severe hearing loss in a patient with Smith-Magenis syndrome. Hum Genet 2001;109:535-541.

13. Chen K-S, Gunaratne PH, Hoheisel JD, Young IG, Miklos GL, Greenberg F et al. The human homologue of the Drosophila melanogaster flightless-I gene (flil) maps within the Smith-Magenis microdeletion critical region in 17p11.2. Am J Hum Genet 1995;56:175-182.

14. Zhao Q, Chen K-S, Bejjani BA, Lupski JR. Cloning, genomic structure, and expression of mouse ring finger protein gene. Znf179. Genomics 1998;49:394-400.

15. Shaffer LG, Kennedy GM, Spikes AS, Lupski JR. Diagnosis of CMT1A duplications and HNPP deletions by interphase FISH: implications for testing in the cytogenetics laboratory. Am J Hum Genet 1997;69:325-331.

16. Lupski JR, and Garcia CA. Charcot-Marie-Tooth peripheral neuropathies and related disorders. In: Scriver CR, Beaudet AL, Sly WS, Valle D, editors. The Metabolic and Molecular Bases of Inherited Disease. New York: McGraw-Hill, 2001:5759-5788.

17. Park JP, Moeschler JB, Davies WS, Patel PI, Mohandas TK. Smith-Magenis syndrome resulting from a de novo direct insertion of proximal $17 \mathrm{q}$ into $17 \mathrm{p} 11.2 . \mathrm{Am} \mathrm{J}$ Med Genet 1998;77:23-27.

18. Potocki L, Chen K-S, Park S-S, Osterholm DE, Withers MA, Kimonis V et al. Molecular mechanism for duplication 17p11.2- the homologous recombination reciprocal of the Smith-Magenis microdeletion. Nat Genet 2000;24:84-87.

19. Madduri NS, Turcich M, Lupski JR, Potocki L. Low adaptive behavior and cognitive functioning in patients with Smith-Magenis syndrome [del(17)(p11.2p11.2)]. Am J Hum Genet 2002;71(suppl):A109.

20. Potocki L, Glaze D, Tan D-X, Park S-S, Kashork CD, Shaffer LG et al. Circadian rhythm abnormalities of melatonin in Smith-Magenis syndrome. J Med Genet 2000; 37:428-433.

21. Smith AC, Gropman AL, Bailey-Wilson JE, Goker-Alpan O, Elsea SH, Blancato J et al. Hypercholesterolemia in children with Smith-Magenis syndrome: del (17)(p11.2p11.2). Genet Med 2002;4:118-125.

22. Schlesinger AE, Potocki L, Poznanski AK, Lupski JR. The hand in Smith-Magenis syndrome (deletion 17p11.2): Evaluation by metacarpophalangeal pattern profile analysis. Pediatr Radiol 2003;33:173-176.

23. Chen RM, Lupski JR, Greenberg F, Lewis RA. Ophthalmic manifestations of SmithMagenis syndrome. Ophthalmology 1996;103:1084-1091.

24. Allanson JE, Greenberg F, Smith AC. The face of Smith-Magenis syndrome: a subjective and objective study. J Med Genet 1999;36:394-397.

25. Sweeney E, Peart I, Tofeig M, Kerr B. Smith-Magenis syndrome and tetralogy of Fallot. J Med Genet 1999;36:501-502.

26. Thomas DG, Jacques SM, Flore LA, Feldman B, Evans MI, Qureshi F. Prenatal diagnosis of Smith-Magenis syndrome (del 17p11.2). Fetal Diagn Ther 2000;15:335-337.

27. Finucane B, Dirrigl KH, Simon EW. Characterization of self-injurious behaviors in children and adults with Smith-Magenis syndrome. Am J Ment Retard 2001;106:52-58.

28. Dykens EM, Smith AC. Distinctiveness and correlates of maladaptive behaviour in children and adolescents with Smith-Magenis syndrome. J Intellect Disabil Res 1998; 42(Pt 6):481-489.

29. De Leersnyder H, De Blois MC, Claustrat B, Romana S, Albrecht U, Von KleistRetzow JC et al. Inversion of the circadian rhythm of melatonin in the SmithMagenis syndrome. J Pediatr 2001;139:111-116.

30. Kleinjan DJ, van Heyningen V. Position effect in human genetic disease. Hum Mol Genet 1998;7:1611-1618. 